\title{
COMPACT INSTALLATION FOR RADIATION PROCESSING OF MATERIALS BY ACCELERATED ELECTRONS
}

\author{
S. Krylov, T. Latypov, G. Mamaev*, S. Mamaev, E. Mirochnik, A. Pirozhenko, \\ S. Poutchkov, I. Seleznev, I. Tenjakov, MRTI RAS, Moscow, Russia \\ A. Korolev, K. Simonov, «Istok», Frjazino, Russia
}

\section{Abstract}

The installation has been designed for processing of articles and materials with the electron beam. The beam is generated by the sealed-off electron gun of unique design and is extracted to the irradiation area through thin Ti foil with $20 \times 10 \mathrm{~cm}^{2}$ area. The electron energy is up to $200 \mathrm{keV}$, pulse beam current - $7 \mathrm{~A}$. The processing is performed in nitrogen. Maximum pulse dose rate is $5 \times 10^{4} \mathrm{kGy} / \mathrm{sec}$. The installation has compact highvoltage power supply, self-contained water cooling, and handy control with the beam parameters regulation within broad ranges. All the installation except the computer is packaged in one case with $2 \times 0.7 \times 0.7 \mathrm{~m}^{3}$ dimensions. The installation has its own radiation shielding and is safe to use: the radiation dose at any place outside the case is near the background radiation level.

\section{INTRODUCTION}

The installation has been designed for processing of the articles and materials with electron beams. The installations of this type can widely be used in research organisations for the investigation of the influence of the radiation on the materials, in clinics for surface radiating sterilisation, and also for other purposes.

Usually, the installations for radiation technology is based on electron accelerators, which work in a continuous current mode and are supplied with devices of linear scanning of the beam. We have the task to design the small, convenient accelerator working in pulse mode. The posed task has required application of the new approaches and original scientific and technical solutions. The electron beam is generated by the sealed off electron gun, which is supplied by the high-voltage pulse transformer, and they both presents joined compact device. The beam is distributed on all area of exposure and has a time structure of short intensive pulses. The installation has a reliable local radiating shielding, it is completely ecologically pure and can be maintained in any location. General parameters are shown in Table 1.

"Work supported in parts by the MRTI RAS, BioSterile Technology, Tukson Inc, Moscow

*E-Mail: mamaev_g@amail.sitek.ru
Table 1: General parameters of the installation

Electron energy (controlled)

Max. pulse power of extracted beam

Irradiation area

Max. dose rate of electron beam

Max. pulse dose rate of electron beam

Max. radiation dose outside the case

Max. power consumed

Overall dimensions (w/o computer)

Overall weight

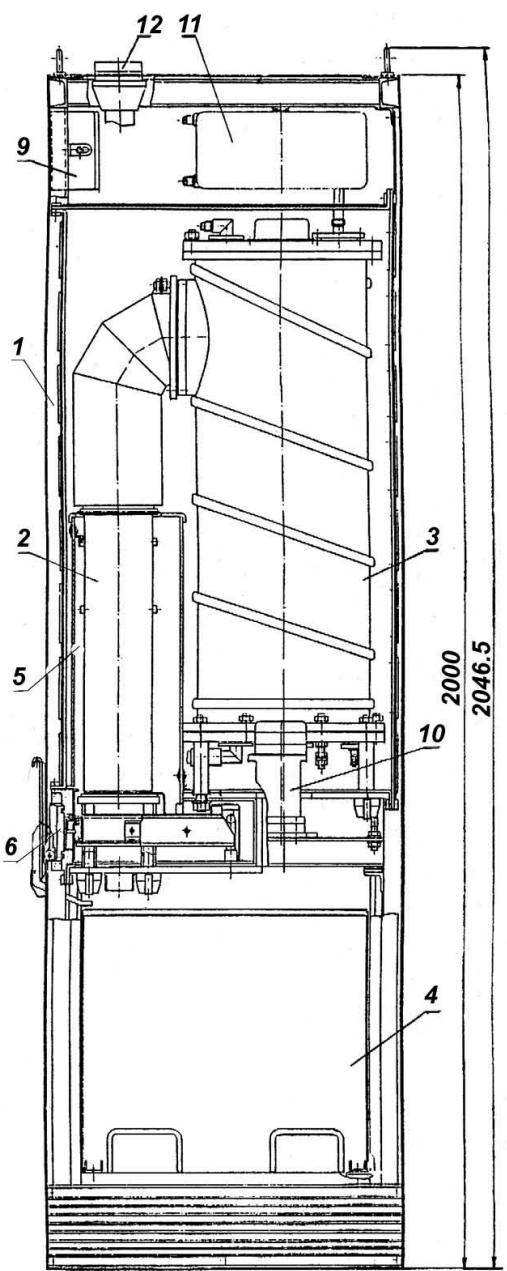

$100 \div 200 \mathrm{keV}$

$300 \mathrm{~kW}$

$200 \times 100 \mathrm{~mm}$

$10 \mathrm{kGy} / \mathrm{sec}$

$5 \times 10^{4} \mathrm{kGy} / \mathrm{sec}$

$0.2 \mathrm{mGy} /$ hour

$2 \mathrm{~kW}$

$2 \times 0.7 \times 0.7 \mathrm{~m}$

$700 \mathrm{~kg}$
Figure 1: The design of installation. 


\section{GENERAL DESCRIPTION}

The design of the installation is shown in Fig. 1, and on the photos - in Fig. 2,3. The main items of the equipment, shown in Fig. 1, designated by numbers from 1 to 6 . Item 1 - case for equipment siting.

\subsection{Electron gun}

Sealed off electronic gun with heated cathode (item 2, Fig. 1) realises generation and extraction of the wide electron beams to the atmosphere. The designed gun is compact and long-lived tube, does not require a system of a vacuum evacuation, has small time of preparation to maintenance, ensures interchangeability and good reproducing of the performances. This gun has a modular construction, which allows receiving a large area of exposure by arrangement of necessary number of guns located closely one to other.

\subsection{High-voltage transformer}

The developed construction of the step-up transformer (item 3, Fig. 1) and its connection with a gun allowed to reach the main purpose - creation of a small-sized construction with a good reliability of working on high voltages. Basic engineering solutions - modular construction of the transformer; high factor of transformation to reduce primary voltage; direct (without a cable) junction with a gun.

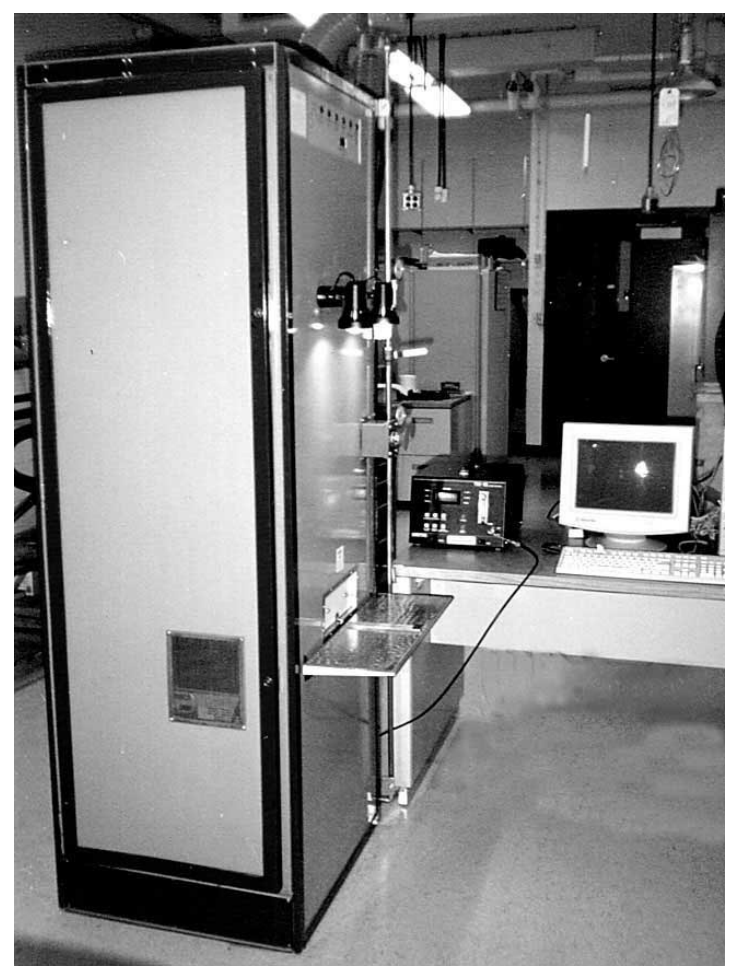

Figure 2: The installation for radiation processing.

The transformer contains 13 identical modules, which are connected in series. Each module contains ring shaped ferromagnetic core, insulating rings mounted on the core; secondary winding wound on the insulating rings; insulating separators between the modules.

The ferromagnetic cores are wound from a thin tape (amorphous alloy). Insulation approximately of 2 microns thickness is applied between the layers of this tape. A thickness of the amorphous tape - 20 microns, induction of saturation - $1.4 \mathrm{~T}, \mathrm{~B}_{\mathrm{r}} / \mathrm{B}_{\mathrm{s}}$ factor -0.9 .

Each module contains two identical secondary windings that are necessary for transmitting electron gun cathode filament current. The transformer has the following parameters:

$\begin{array}{ll}\text { Transformer voltage ratio } & 50 \\ \text { Maximum test output voltage } & 240 \mathrm{kV} \\ \text { Input voltage } & 2 \div 4 \mathrm{kV} \\ \text { Outside core diameter } & 200 \mathrm{~mm} \\ \text { Maximum magnetic field } & 1.4 \mathrm{~T} \\ \text { The tank length } & 840 \mathrm{~mm} \\ \text { The tank diameter } & 300 \mathrm{~mm}\end{array}$

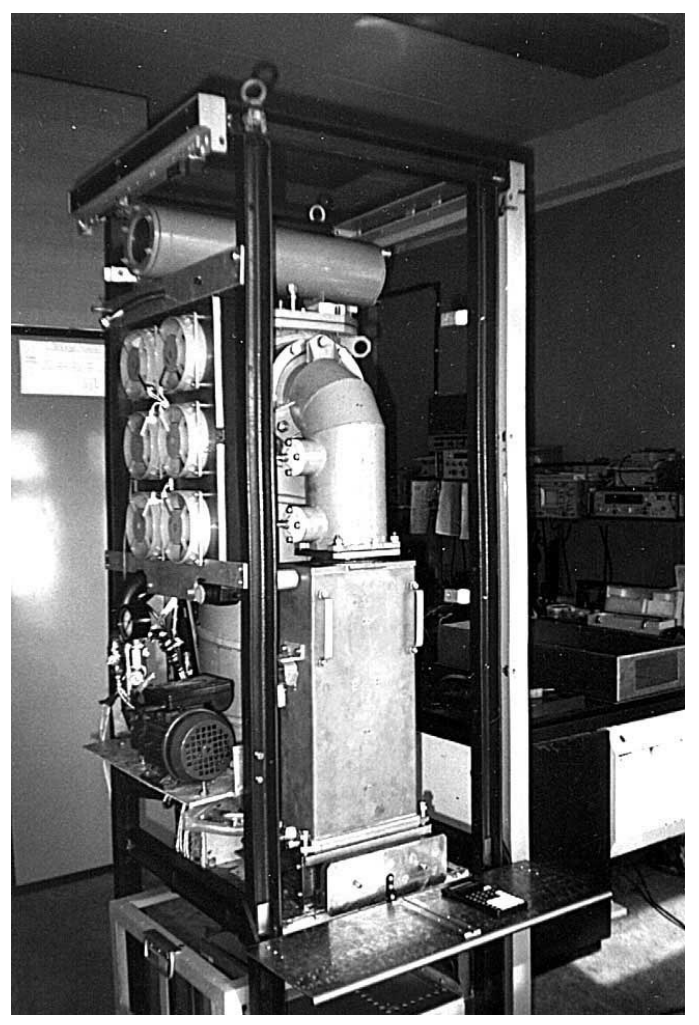

Figure 3: The installation without of a radiation shielding.

\subsection{Pulse modulator}

The modulator (item 4, Fig. 1) generates high-voltage impulses which supplies the input of the step-up transformer. The basic problems of the development of the modulator consist in achieving of the small dimensions, wide control bands of output voltage and pulse repetition rate, good reliability of work. The modulator contains the following main components: 
high-voltage power unit, charging circuit, pulse formation circuit, thyratron switch, measuring voltage dividers.

The high-voltage power unit consists of 6-phase rectifier, filter, high-frequency generator $(30 \mathrm{kHz})$, stepup transformer, and high-voltage rectifier. The unit contains its own control system for protection from overload and high-voltage breakdowns. Its has two feedback circuits, proportional load current and second proportional output voltage. These feedback circuits allow having wide control of the power unit with properly limitation of the charge current. This technical solution excludes the limitation resistors, chokes etc.

The charging circuit is based on the high-voltage thyristor pole. The pulse forming circuit contains a set of capacitors and inductance.

The thyratron switch is assembled using TGP1-1000/25 thyratron that has the following basic parameters: maximum work current of $1000 \mathrm{~A}$ and maximum voltage $25 \mathrm{kV}$. Large reserve in the voltage provides the long operating life of the thyratron, which usually determines the trouble-free time of the modulator.

The modulator is built as a single device enclosed in a casing that serves as an electromagnetic shield designed to reduce the pulse interference directed from the powerful modulator circuits to other installation equipment. The modulator parameters:

Pulse voltage output (controlled

with accuracy better then $3 \%$ )

Pulse duration

2 to $5 \mathrm{kV}$

Pulse repetition rate (controlled) 5 to $150 \mathrm{pps}$

Capacitance of pulse forming circuit

$0.2 \mathrm{mF}$

Overall dimensions of the modulator $\quad 0.65 \times 0.5 \times 0.44 \mathrm{~m}$

\subsection{Radiation shielding}

The basic requirement to a construction of a radiation shielding is the decreasing of the dose at any place near cabinet to a background level $(0.1 \mathrm{mkG} / \mathrm{h})$. The radiation shielding consists of two shells. The first (internal) shell covers the electron gun and irradiation zone. It consists of the set of lead sheets $5 \mathrm{~mm}$ thick with intricate shape. The second (external) shell covers the electron gun, the irradiation zone, and the high-voltage transformer. The shielding allows to decrease radiation in $10^{9}$ times.

The exposure of the samples is produced in the pressure tight camera (item 6, Fig. 1), filled with nitrogen, that eliminates the ozone formation and influence of air oxygen on radiation processing. The shell of the chamber consists of a carriage made of $2 \mathrm{~mm}$ thick stainless steel. Plates of $2 \mathrm{~mm}$ thick lead are fixed to the sides of the carriage. After installing of the carriage, plates were bent to the lead sheet of the base, providing a leek-free joint. Small concentration of oxygen in the chamber was achieved - no more than 20 promille.

\subsection{Control system}

The control of the installation is carried out by means of computer and is automated completely, therefore maintenance of installation can be carried out by not special personal. The computer realises sequential switching on of devices of installation, monitoring of parameters, inclusion of necessary regime and parameters of an exposure. The installation can be operated in three modes: single pulse, continuous, and serial (series of pulses with pauses). The electron energy, pulse repetition rate, duration of irradiation, and the pause intervals may be set within broad ranges. The radiating doze on a surface of an irradiated sample is calculated and shown on the display. The doze may be determined by operator, in this case the necessary time of an exposure is calculated and installed by the computer.

The reliability of safe work with installation is ensured also with system of interlocking, in which there are software and hardware interlocking. For example, the electron beam cannot be switched on, if the carriage removed or poorly entered, and the carriage cannot be removed, when the electron beam is generated.

\section{CONCLUSION}

Such installation is working in one of corporations of USA for more than year. The installation has shown reliable work full ecological safety. 